\title{
Sensitivity of quantitative photoacoustic tomography inversion schemes to experimental uncertainty
}

\author{
Martina Fonseca ${ }^{1}$, Teedah Saratoon ${ }^{1}$, Bajram Zeqiri ${ }^{2}$, Paul Beard ${ }^{1}$, Ben Cox ${ }^{1}$ \\ ${ }^{1}$ Department of Medical Physics and Biomedical Engineering, University College London, \\ Gower Street, London WC1E 6BT, UK; \\ ${ }^{2}$ National Physical Laboratory, Hampton Road, Teddington, Middlesex, TW11 0LW, UK
}

\begin{abstract}
The ability to accurately quantify chromophore concentration from photoacoustic images would have a major impact on pre-clinical and clinical imaging. Recent years have seen significant advances in the theoretical understanding of quantitative photoacoustic imaging and in the development of model-based inversion strategies that overcome issues such as non-uniqueness and non-linearity. Nevertheless, their full in vivo implementation has not successfully been achieved, partially because experimental uncertainties complicate the transition. In this study, a sensitivity analysis is performed to assess the impact on accuracy of having uncertainty in critical experimental parameters such as scattering, beam diameter, beam position and calibration factor. This study was performed using two virtual phantoms, at one illumination and four optical wavelengths. The model-based inversion was applied in 3 variants - one just inverting for chromophores and two others further inverting for either a scaling factor or the scatterer concentration. The performance of these model-based inversions is also compared to linear unmixing strategies - with and without fluence correction. The results show that experimental uncertainties in a priori fixed parameters - especially calibration factor and scatterer concentration - significantly affect accuracy of model-based inversions and therefore measures to ameliorate this uncertainty should be considered. Including a scaling parameter in the inversion appears to improve quantification estimates. Furthermore, even with realistic levels of experimental uncertainty in model-based input parameters, they outperform linear unmixing approaches. If parameter uncertainty is large and has significant impact on accuracy, the parameter can be included as an unknown in model-based schemes.
\end{abstract}

Keywords: quantitative photoacoustic imaging, virtual phantom studies, model-based inversion, unmixing strategies, sources of uncertainty, accuracy

\section{INTRODUCTION}

Photoacoustic Imaging (PAI) measurements have the potential to provide detailed biological information, both spatially due to the high resolution at a wide depth range that acoustic detection enables, and functionally/physiologically due to the high sensitivity of the optical interrogation to different tissue constituents. Nevertheless, the successful quantitative retrieval of physiologically relevant parameters requires dealing with inherent issues such as non-uniqueness, non-linearity and high dimensionality. A general framework for solving the Quantitative Photoacoustic Imaging (qPAI) problem is model-based inversion. This type of framework fully models the light propagation and can therefore provide high accuracy in simulation. ${ }^{1-4}$ The accuracy of these methods does however depend on the validity of the model assumptions, which includes assumptions on the quality of the inputs, often assumed ideal and fully known. In real experimental scenarios though, these inputs are affected by a range of sample-related and setup-related issues. In this study we will assess the effect of uncertainties in the inputs we have least confidence in. Understanding and dealing with these issues is especially important for the successful translation of quantitative PAI frameworks to in vivo pre-clinical and clinical scenarios.

Send correspondence to Martina Fonseca: E-mail: martina.fonseca.13@ucl.ac.uk, Telephone: 44 (0)20 76797886

Photons Plus Ultrasound: Imaging and Sensing 2016, edited by Alexander A. Oraevsky, Lihong V. Wang Proc. of SPIE Vol. 9708, 97084X - (C) 2016 SPIE · CCC code: 1605-7422/16/\$18 · doi: 10.1117/12.2210916 


\subsection{Sources of uncertainty}

Table 1 provides a listing of the assumptions that model-based qPAI procedures rely on. These can, if not satisfied, become sources of error.

Assumption taxonomy

1. Data - we assume:

- the data is complete (i.e. sufficient for exact reconstruction)

- the noise is Gaussian and does not bias the parameter estimates

2. Model Mismatch - we assume the acoustic and optical models accurately capture the essential features of the physics

3. Model Inputs - we assume the inputs are accurate, e.g.:

- optical properties (scattering*, anisotropy, chromophore spectra)

- acoustic properties (sound speed, attenuation)

- Grüneisen parameter*

- source (position*, amplitude*, wavelength)

- sensor (positions, calibration*, angle-and-frequency response)

4. Priors - we may assume the estimated parameters have certain properties, eg. piecewise constant, smooth, homogeneous, time-invariant, etc.

5. Algorithm - we assume the inversion algorithm can find the minimum of the objective function

Table 1: Assumption taxonomy typical of model-based qPAI frameworks. The sources of uncertainty studied in this work are indicated with an asterisk.

In this study we assess in silico how much typical uncertainties on experimental-related input parameters will upset a model-based minimisation. We will focus on the uncertainties affecting the optical inversion that are expected to be the most problematic - scattering behaviour, beam position and diameter, and overall data calibration factor. Acoustic inversion is not addressed since it is not considered to be a major source of uncertainty. ${ }^{1}$ The data is therefore a multiwavelength set of uncalibrated mappings of the initial pressure distribution.

\section{THE VIRTUAL PHANTOM DATA}

Two virtual phantoms are considered - one tube-like and one vessel-like.

\subsection{Tube phantom}

The first virtual phantom replicates an experimental phantom design. The domain is $60 \times 60 \mathrm{~mm}$, defined by $N h=3721$ nodes and $N e=7200$ regular triangular elements. 4 tubes with $600 \mu$ m diameter are present. The domain being $2 \mathrm{D}$, the tubes are represented as their circular cross-section. The true chromophore and scatterer concentration distributions employed can be seen in Figure 1 (a). Copper chloride $\mathrm{CuCl}_{2}$ and nickel chloride $\mathrm{NiCl}_{2}$ were used as the representative chromophores, given their previously reported suitability for qPAI studies. ${ }^{5}$ Considering the tubes from top left to bottom right, the concentration of $\mathrm{CuCl}_{2}$ was $c_{\mathrm{CuCl}_{2}}=\{0.25,0.75,1,0\}$, and the concentration of $\mathrm{NiCl}_{2}$ was $c_{\mathrm{NiCl}_{2}}=\{0.75,0.25,0,1\}$, defined as a fraction of the concentrations of the respective mother batches $c_{C u M}=35.5069 \mathrm{~g} / \mathrm{l}$ and $c_{N i M}=399.783 \mathrm{~g} / \mathrm{l}$. Values were chosen such that

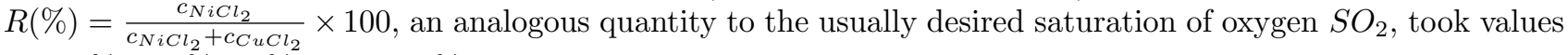
of $75 \%, 25 \%, 0 \%$ and $100 \%$. The background was such that $c_{\mathrm{NiCl}_{2}, b k g}=0.005, c_{\mathrm{CuCl}_{2}, b \mathrm{~kg}}=0.005$ and $R=50 \%$. Water $\mathrm{H}_{2} \mathrm{O}$, the solvent, is the additional chromophore that is present and known in the entire domain. Spectra for $\mathrm{CuCl}_{2}, \mathrm{NiCl}_{2}$ and $\mathrm{H}_{2} \mathrm{O}$ are given in Figure 1 (b). Reduced scattering $\mu_{s}^{\prime}$ was set to what is expected in a $1 \%$ intralipid solution, ${ }^{6}$ the typical concentration when tissue scattering properties are to be mimicked $\left(\sim 1 \mathrm{~mm}^{-1}\right)$, and for simplicity was assumed to be spatially constant in concentration $\left(a_{1}(x, y)=1\right)$. 


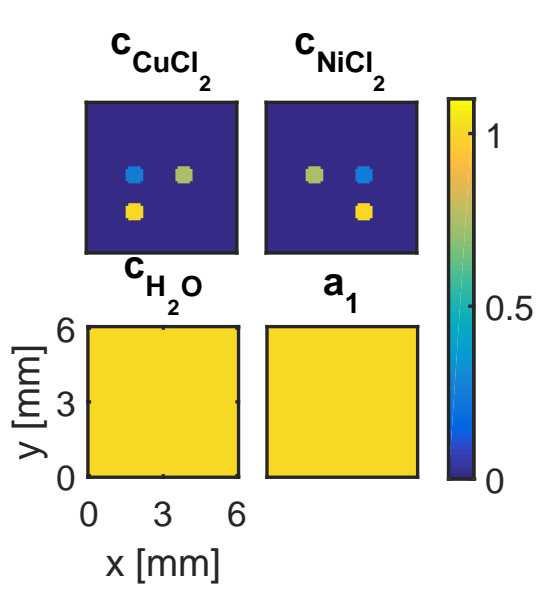

(a)

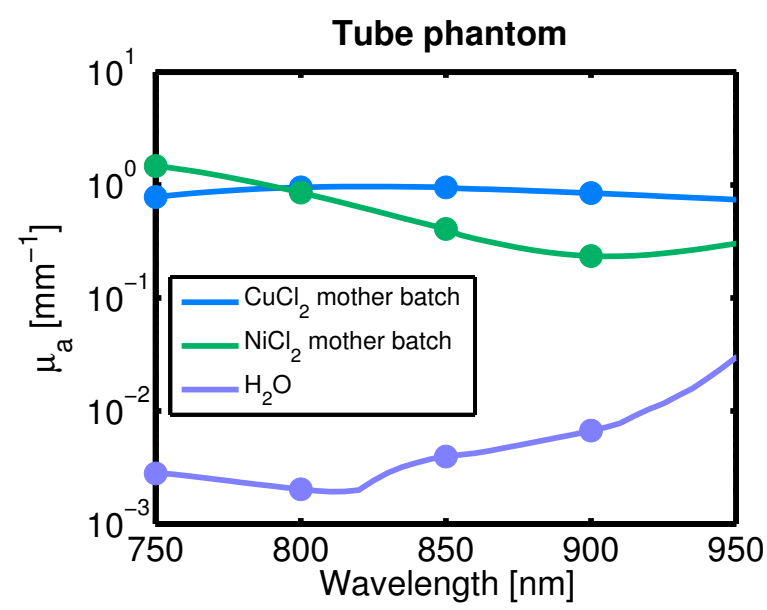

(b)

Figure 1: (a) Distribution and concentration of the chromophores $\left(c_{k}\right)$ and scatterer $\left(a_{1}\right)$ of interest for the tube phantom. Concentration units for each constituent are given in respect to its respective reference spectrum. (b) Reference absorption spectra for the chromophores present in the domain. $\mathrm{CuCl}_{2}$ and $\mathrm{NiCl}_{2}$ were measured experimentally in a spectrophotometer, whilst values for water were taken from the literature. ${ }^{7}$

\subsection{Vessel phantom}

The second phantom has a domain with the same dimensions as the tube phantom but aims to emulate an in vivo scenario, being constituted primarily by two vessels, both with hemoglobin concentration $c_{H b T}=150$ $\mathrm{gl}^{-1} \simeq 2.327 \mathrm{mM}$, typical for human blood. ${ }^{8}$ The true chromophore and scatterer concentration distributions are shown in Figure 2 (a). The left and right vessel were assigned oxygen saturation levels $\mathrm{SO}_{2}=90 \%$ and $\mathrm{SO}_{2}=70 \%$, in the typical range for arterial and venous blood respectively. ${ }^{9}$ Water was present as an additional chromophore, at a fractional volume of $W=0.55$ inside the vessels, which is a typical fraction for the plasma component of blood (plasma itself is mainly constituted by water). ${ }^{9}$ The background properties were chosen to match abdominal tissue, such that $c_{H b T}=0.0125 \mathrm{mM} \simeq 0.8057 \mathrm{gl}^{-1}$ and $S O_{2}=76 \%$, with water volume fraction $W=0.11$ and fat volume fraction $F=0.69 .^{8,10}$ For the inversion, $c_{\mathrm{HbO}_{2}}$ and $c_{\mathrm{Hb}}$ were the unknowns, with $c_{\mathrm{H}_{2} \mathrm{O}}$ and $c_{f a t}$ known throughout the domain. Spectra for $\mathrm{HbO}_{2}, \mathrm{Hb}$ and $\mathrm{H}_{2} \mathrm{O}$ and fat are given in Figure 2 (b). Unlike the first phantom, in this case scattering was spatially varying. The vessel areas were assigned scattering typical of whole blood $^{8,11}$ and the background was assigned a value typical for subcutaneous-adipose tissue, ${ }^{8,12}$ $a_{2,(\text { vessels })}=2.2$ and $a_{2, \text { (background })}=1.54$ where $\mu_{s}^{\prime}=a_{2} \frac{\lambda}{500 \mathrm{~nm}}^{-0.68}$. The power law values for whole blood and adipose tissue are similar enough ( $b=0.66$ and $b=0.68$ respectively) therefore a common value of 0.68 was used as a first approximation for simplicity. 


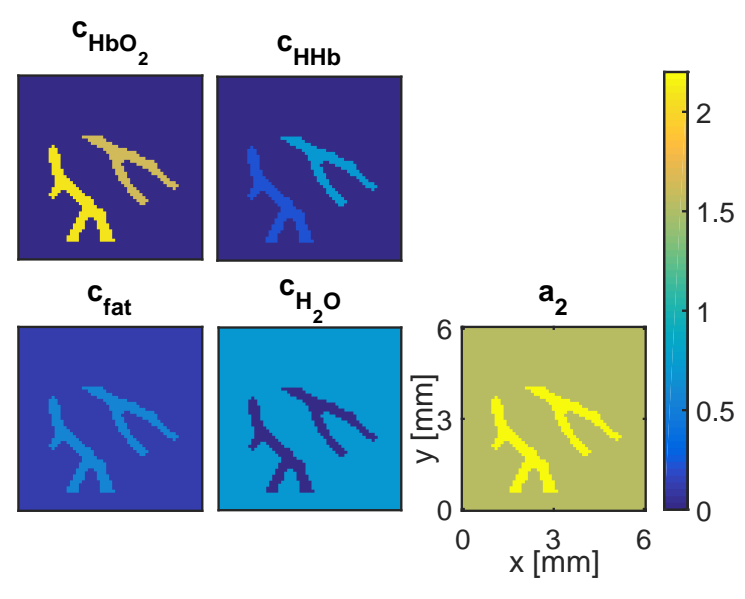

(a)

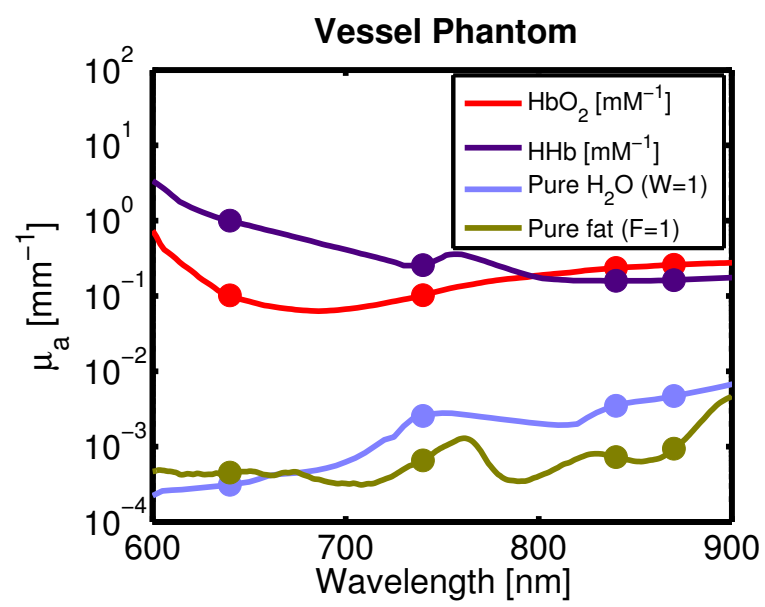

(b)

Figure 2: (a) Distribution and concentration of the chromophores $\left(c_{k}\right)$ and scatterers $\left(a_{2}\right)$ of interest for the vessel phantom. Concentration units for each constituent are given relative to its respective reference spectrum. (b) Reference absorption spectra for the chromophores present in the domain. Values were taken from the literature - water, ${ }^{7}$ hemoglobin, ${ }^{13}$ fat. ${ }^{14,15}$

\subsection{Light Model}

The light model employed to obtain the 'measured' data was the $2 \mathrm{D}$ diffusion approximation ${ }^{16}$ (for both phantoms, the background was such that $\mu_{s}^{\prime}>>\mu_{a}$, and the structures of interest were positioned at a minimum depth $d$ from the source such that the condition $d>>\frac{1}{\mu_{t}}$ was respected by having $d$ be greater than $\frac{1}{\mu_{t}}$ by at least a five-fold factor, where $\mu_{t}=\mu_{s}+\mu_{a}$ is the transport coefficient. $\left.{ }^{1}\right)$. Four wavelengths $(L=4)$ were considered $750,800,850$ and $900 \mathrm{~nm}$ for the tube phantom and 640,740, 840 and $870 \mathrm{~nm}$ for the vessel phantom. The illumination source was defined as a top hat beam located at the top of the domain, $Q_{\text {pos }}=\left(x_{Q}, y_{Q}\right)=([0.55 .5], 6)$ $\mathrm{mm}$ (diameter $Q_{\text {diam }}=5 \mathrm{~mm}$ ).

The 'measured' data that will be used as starting point for the inversions is the uncalibrated initial pressure distribution, defined as $p_{0, \text { meas }}=K_{\text {true }} \mu_{a} \Phi$, or in matrix notation for a single nodal point: ${ }^{17}$

$$
\left[\begin{array}{c}
p_{0}\left(\lambda_{1}\right) \\
p_{0}\left(\lambda_{2}\right) \\
p_{0}\left(\lambda_{3}\right) \\
p_{0}\left(\lambda_{4}\right)
\end{array}\right]=K_{\text {true }}\left[\begin{array}{ccc}
\Phi\left(\lambda_{1}\right) & \cdots & 0 \\
\vdots & \ddots & \vdots \\
0 & \cdots & \Phi\left(\lambda_{4}\right)
\end{array}\right]\left[\begin{array}{cccc}
\alpha_{c_{1}}\left(\lambda_{1}\right) & \alpha_{c_{2}}\left(\lambda_{1}\right) & \cdots & \alpha_{c_{M}}\left(\lambda_{1}\right) \\
\alpha_{c_{1}}\left(\lambda_{2}\right) & \alpha_{c_{2}}\left(\lambda_{2}\right) & \cdots & \alpha_{c_{M}}\left(\lambda_{2}\right) \\
\alpha_{c_{1}}\left(\lambda_{3}\right) & \alpha_{c_{2}}\left(\lambda_{3}\right) & \cdots & \alpha_{c_{M}}\left(\lambda_{3}\right) \\
\alpha_{c_{1}}\left(\lambda_{4}\right) & \alpha_{c_{2}}\left(\lambda_{4}\right) & \cdots & \alpha_{c_{M}}\left(\lambda_{4}\right)
\end{array}\right]\left[\begin{array}{c}
c_{1} \\
c_{2} \\
\vdots \\
c_{M}
\end{array}\right]
$$

where $\Phi\left(\lambda_{l}\right)$ is the fluence at wavelength $l, \alpha_{c_{m}}\left(\lambda_{l}\right)$ is the reference absorption spectrum for chromophore $m$ at wavelength $l, M$ is the total number of chromophores and $K_{\text {true }}=1$ is a spatially-constant factor that encompasses thermoelastic efficiency $\Gamma$ and acoustic sensor response. This means the data is considered to be in arbitrary units rather than units of pressure or absorbed energy density, which is typical of what would be obtained experimentally on a system with uncalibrated detectors.

Figure 3 shows the absorption $\mu_{a}$, the fluence $\Phi$ and the absorbed energy density $H=\mu_{a} \Phi$ for the tube phantom, at the four wavelengths of interest. 

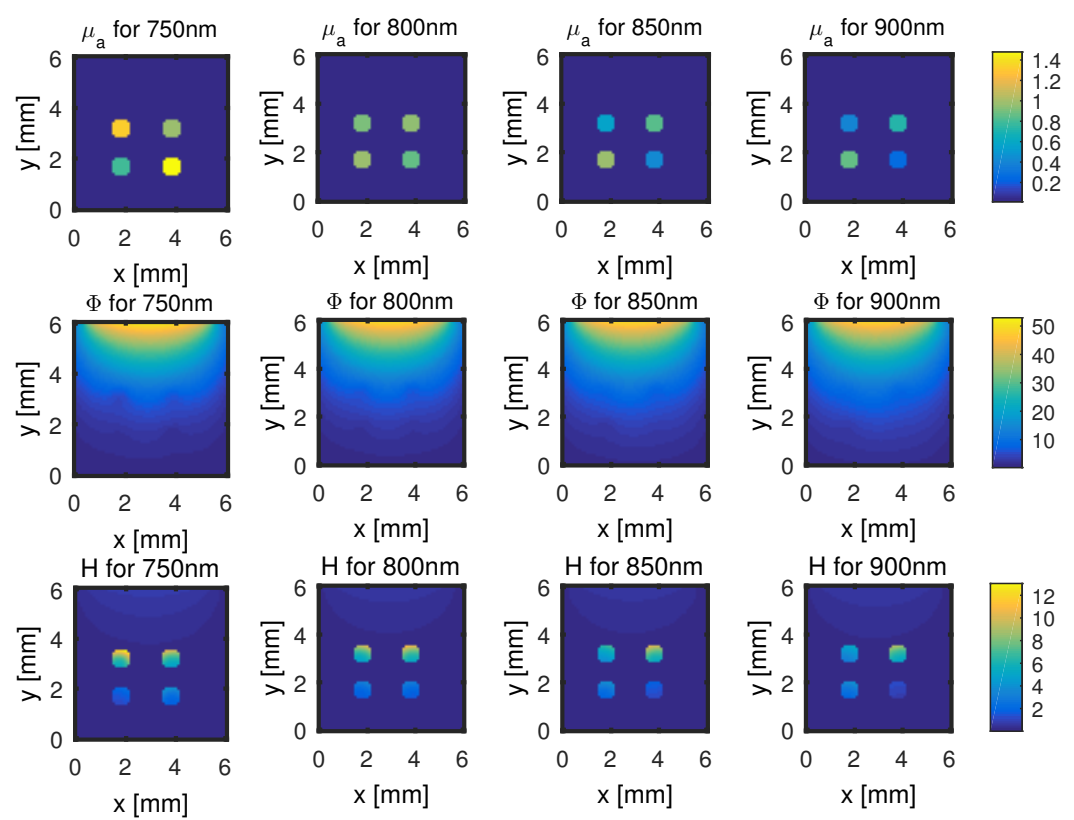

Figure 3: [Tube phantom] True absorption coefficient $\left(\mu_{a}\right)$, true fluence $(\Phi)$ and true absorbed energy $(H)$ at wavelengths $750,800,850$ and $900 \mathrm{~nm}$.

\section{THE INVERSION SCHEMES}

In this study, the main aim of the qPAI strategies is to retrieve the concentration of the chromophores of interest $\left(c_{\mathrm{NiCl}_{2}}\right.$ and $c_{\mathrm{CuCl}_{2}}$ for the tube phantom and $c_{\mathrm{HbO}_{2}}$ and $c_{\mathrm{Hb}}$ for the vessel phantom) from multiple-wavelength and single illumination data, or alternatively to retrieve an image ratio related parameter analogous to oxygen saturation, $R$.

\subsection{Model-based inversion strategies}

For the model-based inversion, a gradient-based minimisation strategy is employed to retrieve $\mathbf{c}_{\mathbf{k}}$ from the uncalibrated initial pressure maps, where the forward light model is given by the $2 \mathrm{D}$ light diffusion approximation equation. ${ }^{16}$ The acoustic problem is assumed to be perfectly solved up to a constant factor $K$. The error functional is defined as:

$$
\varepsilon=\sum_{l=1}^{L} \sum_{h=1}^{N h}\left[p_{0, \text { meas }_{h}}\left(\lambda_{l}\right)-p_{0, \text { sim }_{h}}\left(\mathbf{u}, \lambda_{l}\right)\right]^{2}
$$

where $p_{0, \text { meas }}\left(\lambda_{l}\right)$ is the 'measured' uncalibrated initial pressure distribution and $p_{0, \text { sim }}\left(\mathbf{u}, \lambda_{l}\right)$ is the estimated uncalibrated initial pressure distribution for a given iteration with estimates $\mathbf{u}$ for the unknowns, where subscripts $l$ and $h$ denote different wavelengths and nodal positions respectively. The minimisation algorithm chosen is the quasi-Newton limited-memory BFGS (L-BFGS). ${ }^{18}$ The functional gradients $\frac{\partial \varepsilon}{\partial c_{k}}$ and $\frac{\partial \varepsilon}{\partial a}$ are calculated with the aid of the adjoint method. ${ }^{19,20}$ It is important to note that this study is independent of the particular choice of minimisation algorithm, and L-BFGS could have been replaced with any other algorithm that similarly found the global minimum of the cost function. It is the degree to which the uncertainties in the experimental parameters move the minimum in the error functional (and hence the estimated quantities) that is important in this study, and not the particular algorithm used to locate it.

Three different inversions were performed:

- L-BFGS- $\left(\hat{\mathbf{c}_{\mathbf{k}}}\right)$ - estimates both chromophores $\mathbf{c}_{\mathbf{k}}$ from single-illumination and $\mathrm{L}=4$-wavelength data, which gives a number of unknowns $N_{\text {unknowns }}=2 \times N h=7442$. 
- L-BFGS- $(\hat{\mathbf{c}}, \hat{K})$ - estimates $\mathbf{c}_{\mathbf{k}}$ and the overall calibration factor $K$ (a factor that encompasses thermoelastic efficiency $\Gamma$, mechano-electrical coupling) from single-illumination and 4 -wavelength data. $N_{\text {unknowns }}=$ $2 \times N h+1=7443$.

- L-BFGS- $\left(\hat{\mathbf{c}_{\mathbf{k}}}, \hat{\mathbf{a}}\right)$ estimates $\mathbf{c}_{\mathbf{k}}$ and the scatterer spatial distribution a from single-illumination and 4wavelength data. $N_{\text {unknowns }}=3 \times N h=11163$. The spectral signature of the scatterer is assumed known.

All simulations were allowed to run till the difference in the error functional from one iteration to the next was less than $10^{-6}$ or otherwise for a maximum of 1000 iterations. Given the 1.5 fold increase in unknowns for the L-BFGS- $\left(\hat{\mathbf{c}_{\mathbf{k}}}, \hat{\mathbf{a}}\right)$ case, the maximum number of iterations was 1500 in this case.

Simulations were run from 2 different starting values - the first initialisation was the unperturbed groundtruth (sufficiently close to the minimum in the perturbed case to ensure that this global minimum was found) and the second initialisation was set at $90 \%$ of the true background values (in all cases this converged to the same minimum).

\subsection{Unmixing strategies}

Linear unmixing strategies have become popular for in vivo applications due to their efficiency, speed and ease of implementation. These rely on different assumptions - e.g. direct linear unmixing assumes there is no spectral corruption or fluence wavelength dependence at each spatial point, ${ }^{21,22}$ linear unmixing with $1 \mathrm{D}$ attenuation compensation assumes light decays diffusely and uni-dimensionally by a fixed background attenuation level, ${ }^{22}$ independent component analysis (ICA) assumes all components of interest to be extracted are independent and linearly separable. ${ }^{21}$ Since the previous assumptions are rarely wholly true, only limited accuracy can be achieved with these strategies, and under specific conditions.

Estimates from linear unmixing, which assumes that $\Phi\left(\lambda_{l}\right)=\Phi\left(\lambda_{j}\right)=\gamma, \forall l, j \in L, \gamma$ constant, were compared to the perturbed estimates using model-based inversion. The linear unmixing scheme used was (defined for each nodal position):

$$
\left[\begin{array}{l}
K^{\prime} c_{1} \\
K^{\prime} c_{2}
\end{array}\right] \approx\left[\begin{array}{ll}
\alpha_{c_{1}}\left(\lambda_{1}\right) & \alpha_{c_{2}}\left(\lambda_{1}\right) \\
\alpha_{c_{1}}\left(\lambda_{2}\right) & \alpha_{c_{2}}\left(\lambda_{2}\right) \\
\alpha_{c_{1}}\left(\lambda_{3}\right) & \alpha_{c_{2}}\left(\lambda_{3}\right) \\
\alpha_{c_{1}}\left(\lambda_{4}\right) & \alpha_{c_{2}}\left(\lambda_{4}\right)
\end{array}\right]^{\dagger}\left[\begin{array}{l}
p_{0}\left(\lambda_{1}\right) \\
p_{0}\left(\lambda_{2}\right) \\
p_{0}\left(\lambda_{3}\right) \\
p_{0}\left(\lambda_{4}\right)
\end{array}\right]
$$

where $K^{\prime}=K \gamma$ is the adapted calibration factor accounting for light fluence, $\dagger$ denotes the pseudo-inverse and $c_{1}$ and $c_{2}$ are the chromophores of interest $\left(\mathrm{CuCl}_{2}\right.$ and $\mathrm{NiCl}_{2}$ for the tube phantom and $\mathrm{HbO}_{2}$ and $\mathrm{Hb}$ for the vessel phantom), with contribution from any remaining chromophores assumed negligible since their absorption is orders of magnitude lower. An estimate of the relative concentration $R$ was then found as:

$$
R=\frac{K^{\prime} c_{2}}{K^{\prime} c_{1}+K^{\prime} c_{2}}=\frac{c_{2}}{c_{1}+c_{2}}
$$

where unknown $K^{\prime}$ cancels out.

Linear unmixing was performed both without $(\mathrm{LU})$ and with $\left(\mathrm{LU}_{C}\right)$ a simple fluence correction $\left(\Phi_{\text {approx }}=\right.$ $\left.e^{-\mu_{f i t}\left(y_{Q}-y\right)} \forall x\right)$ being applied to $p_{0}$, where the value $\mu_{f i t}$ was obtained through an exponential fit to the laterally $\left(x\right.$-wise) integrated background signal decay ${ }^{22}$.

\section{SENSITIVITY ANALYSIS AND PERFORMANCE COMPARISON OF THE INVERSION SCHEMES}

The following scenarios of potential experimental-related error were tested:

- Error in the assignment of the calibration factor $K, \varepsilon(K)$; 
- Error in the assignment of the overall scattering coefficient $\mu_{s}^{\prime}, \varepsilon\left(\mu_{s}^{\prime}\right)$;

- Error in the assignment of the horizontal positioning of the beam $Q_{\text {pos }}, \varepsilon\left(Q_{\text {pos }}\right):=\varepsilon\left(x_{Q}\right)$;

- Error in the assignment of the beam diameter $Q_{\text {diam }}, \varepsilon\left(Q_{\text {diam }}\right)$;

Errors in the qPAI strategies were computed with the following metrics:

- Relative error in $\mathbf{c}_{\mathbf{k}}$ estimation: $R E\left(\mathbf{c}_{\mathbf{k}}\right)=\frac{\left\|\hat{\mathbf{c}_{\mathbf{k}}}-\mathbf{c}_{\mathbf{k}, \text { true }}\right\|}{\left\|\mathbf{c}_{\mathbf{k}, \text { true }}\right\|} \times 100$;

- Relative error in a estimation: $R E(\mathbf{a})=\frac{\left\|\hat{\mathbf{a}}-\mathbf{a}_{\text {true }}\right\|}{\| \mathbf{a} \text { true } \|} \times 100$;

- Relative error in intra-luminal $\mathbf{R}^{\mathrm{IL}}$ estimation: $R E\left(\mathbf{R}^{I L}\right)=\frac{\left\|\hat{\mathbf{R}}^{I L}-\mathbf{R}_{\text {true }}{ }^{I L}\right\|}{\left\|\mathbf{R}_{\text {true }}{ }^{I L}\right\|} \times 100$;

where \|\| denotes the euclidean norm and $\mathbf{R}^{I L}$ is defined: intra-tube for the tube phantom, $\mathbf{R}^{\text {tubes }}(\%)=$ $\frac{\mathbf{c}_{\mathrm{NiCl}_{2}}^{\text {tubes }}}{\mathbf{c}_{\mathrm{NiCl} l_{2}}^{\text {tubes }}+\mathbf{c}_{\mathrm{CuCl}}^{\text {tubes }}} \mathrm{Cu}$, and intra-vessel for the vessel phantom $\mathbf{R}^{\text {vessels }}(\%)=S O_{2}^{\text {vessels }}(\%)=\frac{\mathbf{c}_{\mathrm{HbO}}^{\text {vessels }}}{\mathbf{c}_{\mathrm{HbO}}^{\text {vessels }}+\mathbf{c}_{\mathrm{Hb}}^{\text {vessels }}} \times 100$.

Simulations with both types of initialisation, when convergent, did yield the same value, meaning that the results were independent of the starting estimates within reasonable values and that they give a correct depiction of the shift of the global minimum of the error functional when a perturbation (parameter uncertainty) is introduced.

\subsection{Uncertainty on overall calibration factor $\mathrm{K}$}

The parameter $K$ is a scalar calibration factor that accounts for the Grüneisen parameter, for the incident fluence and for the mechano-electrical coupling of the transducer, relating the absorbed energy density mapping to the actual measured image - an uncalibrated initial pressure image. $K$ is assumed constant here, though the various factors above could have a spatially-dependent variation, or even frequency-dependent in the case of the mechano-electrical coupling. Unless a thorough calibration of $K$ is performed, or unless the value of interest is explicitly set as a ratio of two images from the start, errors in defining $K$ will affect qPAI strategies. Here, $K$ is allowed to vary between $\varepsilon(K)=\{-50,-40,-30,-20,-10,0,10,20,30,40,50\} \%$, meaning from $\mathrm{K}=0.5$ to 1.5 .

The effect of this error in both phantoms is tested for the $\left(\hat{\mathbf{c}_{\mathbf{k}}}\right)$ algorithm, as well as for the self-calibrating $\left(\hat{\mathbf{c}_{\mathbf{k}}}, \hat{K}\right)$ algorithm. As seen in Figure 4 (a), erroneous estimation of a fixed $K$ greatly affects the error in $\mathbf{c}_{\mathbf{k}}$ estimation for $\left(\hat{\mathbf{c}_{\mathbf{k}}}\right)$. On the other hand, as expected, $\left(\hat{\mathbf{c}_{\mathbf{k}}}, \hat{K}\right)$ manages to correctly estimate both $K$ and $\hat{\mathbf{c}}$ from any initial erroneous $K$ estimate since the problem is well-posed. This does come at the expense of a slower convergence compared to when $K$ is well-known and fixed (data not shown).

When looking at the intra-tube error for the saturation-equivalent parameter $\mathbf{R}^{\text {tubes }}$ in the tube phantom (Figure 4 (b)), though the error is lower than that for $\mathbf{c}_{\mathbf{k}}$ it is still quite prominent. For the ratio to successfully cancel $K$ the images would have needed to be divided before entering the inversion pipeline, not after the estimation. Even so, the comparison shows that for uncertainty in $K$ up to $40 \%$, ( $\hat{\mathbf{c}}$ ) still performs better than linear unmixing strategies. Data for $\mathbf{R}^{\text {vessels }}$ is omitted for clarity throughout the paper. Nevertheless, for all uncertainty cases studied, the trends observed were similar to those for $\mathbf{R}^{\text {tubes }}$, though at a lower absolute error, which is thought to be due to the fact that the difference in $R$ between background and vessels is not as significant as the one seen between background and tubes in the tube phantom, resulting in less spectral corruption. 


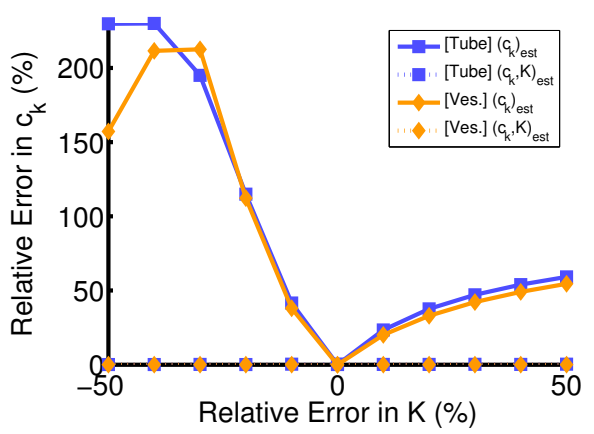

(a)

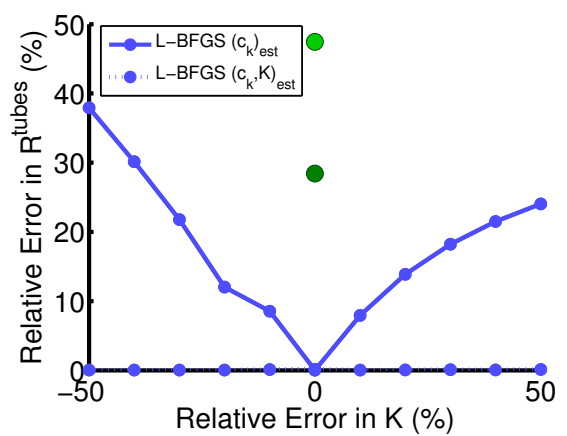

(b)

Figure 4: (a) Relative error in chromophore concentration vs error in calibration factor $K$. (b) [Tube phantom] Relative error in intra-tube $R$ vs error in calibration factor $K$. LU - light green; $\mathrm{LU}_{C}$ - dark green.

\subsection{Uncertainty on reduced scattering coefficient}

The scattering coefficient distribution is typically unknown. Estimating it simultaneously with $\mu_{a}$ distribution is not possible due to non-uniqueness. Multiple-illumination strategies can overcome this, ${ }^{23}$ but involve more complex experimental designs. Alternatives that have been proposed for single-illumination involve either setting the scattering to an estimate of its true overall or background value, or estimating scattering at multiple wavelengths by incorporating prior information on its spectral behaviour. ${ }^{24}$

To assess the effect that having uncertainty in the reduced scattering coefficient has on the latter two approaches, the reduced scattering coefficient was varied by a constant multiplicative factor, meaning that the relative wavelength-dependence was always maintained, but the concentration of the scatterer at each point $\mathbf{a}(\mathbf{x}, \mathbf{y})$ was altered: $\varepsilon\left(\mu_{s}^{\prime}\right)=\varepsilon\left(\mu_{s}\right)=\varepsilon(a)=\{-50,-40,-30,-20,-10,0,10,20,30,40,50\} \%$. 


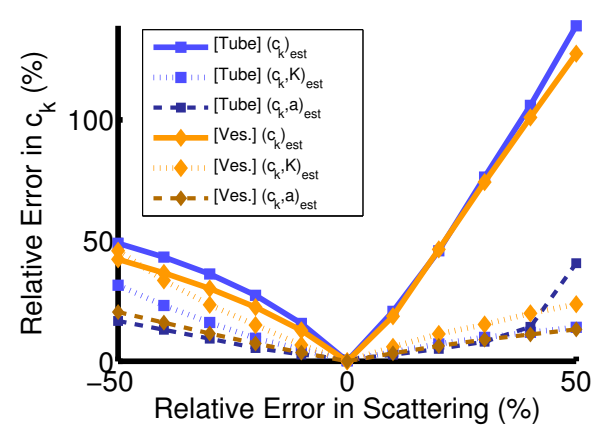

(a)

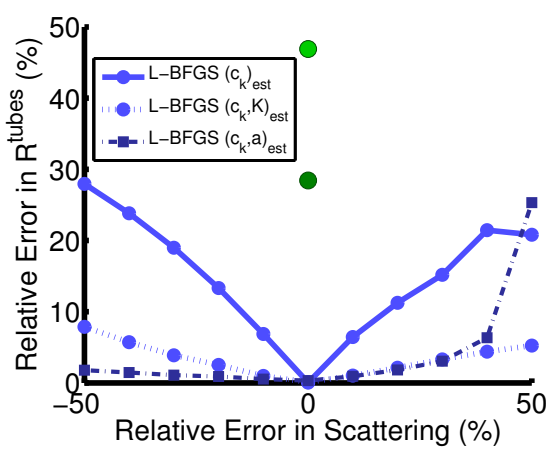

(b)

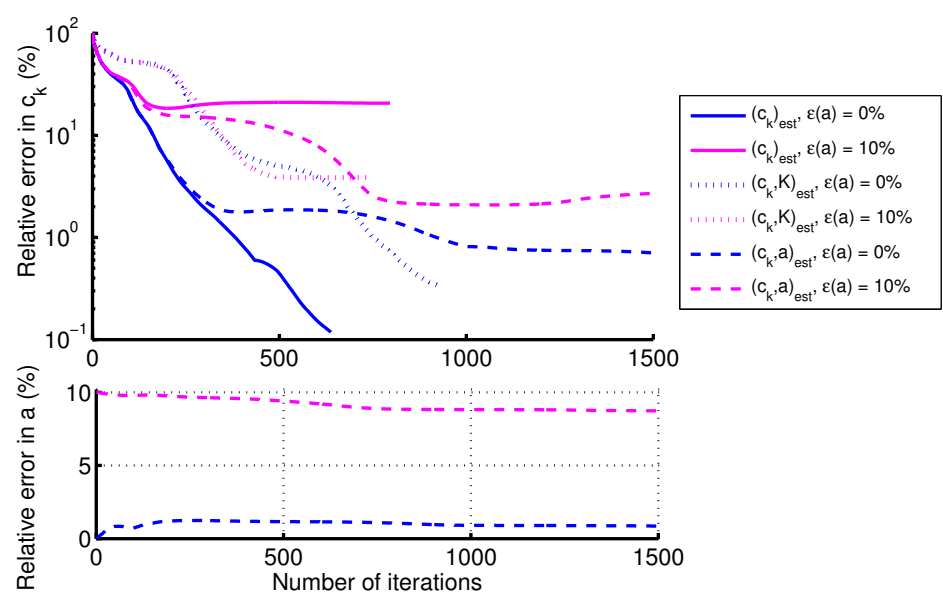

(c)

Figure 5: (a) Relative error in chromophore concentration vs error in scatterer concentration $a$. (b) [Tube phantom] Relative error in intra-tube $R$ vs error in scattering. LU - light green; $L_{C}$ - dark green. (c) [Tube phantom] Relative error in chromophore concentration (top) and scatterer concentration (bottom) vs number of iterations.

For both phantoms, errors in the assumed scattering coefficient led to considerable error in $\mathbf{c}_{\mathbf{k}}$ estimation by $\left(\hat{\mathbf{c}_{\mathbf{k}}}\right)$, especially when scattering was assumed to be higher than its true value: a $50 \%$ over-estimation of scattering led to errors up to $140 \%$ (Figure 5 (a) ). The self-calibrating algorithm, $(\hat{\mathbf{c}}, \hat{K})$, led to considerable improvement in the estimation, though as seen in Figure 5 (c) for the $\varepsilon(a)=10 \%$ case, improvement in $\mathbf{c}_{\mathbf{k}}$ estimation still eventually stabilises at a non ground-truth local minimum (dotted pink line). The fact that the estimation is seen to stabilise at this local minimum and does not further converge to the ground-truth is expected, since adding one free floating spatially-constant parameter $K$ is not enough for the model to fully explain disparities caused by a widespread change in scattering which will affect fluence and $p_{0}$ both intensity-wise and spatially-wise. Nonetheless, though $K$ not expected to be able to explain the spatially dependent error, it seems it can help mitigate/absorb the average error in the initial pressure intensity, which is why the local minimum that is reached is an improvement on the local minimum of $\left(\hat{\mathbf{c}_{\mathbf{k}}}\right)$. For further comparison, the $\left(\hat{\mathbf{\mathbf { k } _ { \mathbf { k } }}}, \hat{\mathbf{a}}\right)$ algorithm, which minimises for the spatially-dependent scatterer concentration with a known wavelength behaviour, was employed. In theory $\left(\hat{\mathbf{c}_{\mathbf{k}}}, \hat{\mathbf{a}}\right)$ should be able to converge to the true minimum since the problem is unique and fully explains the data. In practice it does not within the 1500 iterations due to slow convergence and poor sensitivity to scattering away from the source. Despite that, for both phantoms this algorithm performs the best out the the three, as expected. It does not improve that considerably compared to $(\hat{\mathbf{c}}, \hat{K})$ though, which is note-worthy since $(\hat{\mathbf{c}}, \hat{\mathbf{a}})$ is already more memory-intensive ( $\sim 1.5$ more unknowns), and needs a longer computation time per iteration. Figure 6 further illustrates, for the tube phantom, how accurate the three inversions are for $\varepsilon(\mathbf{a})=-50 \%$, compared to the ground-truth. Note how for $(\hat{\mathbf{k}}, \hat{\mathbf{a}})$ improvement on estimated $\mathbf{a}$ is significant mainly close to the source.

Error in the estimated $\mathbf{R}^{\text {tubes }}$ shows the performance of the 3 model-based inversions follows the same 
tendency as the error in estimated $\hat{\mathbf{k}}$, but with lower overall error. As for the comparison with the linear unmixing frameworks, model-based inversions always perform better, except for $\left(\hat{\mathbf{c}_{\mathbf{k}}}\right)$ when scattering is underestimated by more than $40 \%$ (Figure 5 (b) - tube phantom ) .

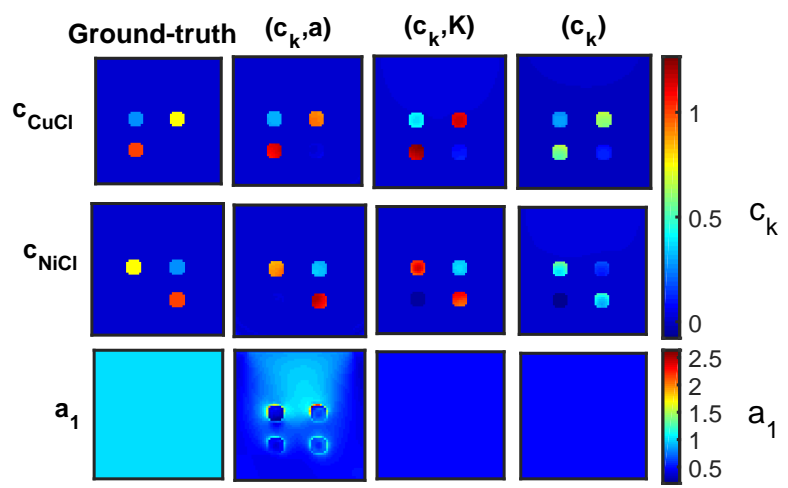

Figure 6: [Tube phantom] Comparison of outcome between inversions with wrong scattering estimate $a$. First column

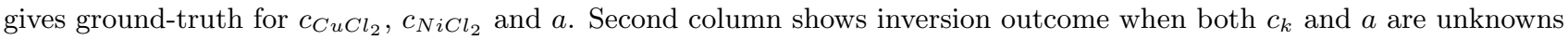
to be inverted, and $a_{0}=50 \% a_{t r u e}$. Third and fourth columns show outcome for L-BFGS- $\left(c_{k}, K\right)$ and L-BFGS- $\left(c_{k}, a\right)$ respectively, where scattering is fixed as $a=50 \% a_{\text {true }}$.

\subsection{Uncertainty on excitation beam}

Beam profile $Q$ is usually assumed known in qPAI inversion strategies. Nevertheless, that is not always the case. Uncertainties in positioning, intensity, diameter and profile are often present, and these may even vary with wavelength. Pre-acquisition steps can be taken to map the beam profile approximately - for instance by probing a black absorber -, nevertheless this will still be an approximation and will especially not correctly describe the incidence on the surface of most in vivo subjects of interest, which will be non-flat. Here, the impact of errors in beam positioning $Q_{p o s}$ and beam diameter $Q_{\text {diam }}$ is described. The beam is assumed to be top hat (collimated) in all instances.

\subsubsection{Uncertainty on beam positioning}

The beam positioning was varied from its central position to its most leftward and rightward possible position in the domain, at incremental steps. More specifically, $\delta\left(Q_{\text {pos }}\right)=\{-10,-8,-6,-4,-2,0,2,4,6,8,10\} \% Q_{\text {diam }}$. Figure 7 (a) shows that changing positioning led to an expected error in optical property estimation with $\left(\hat{\mathbf{c}_{\mathbf{k}}}\right)$, nevertheless the error did not exceed $14 \%$ for a $10 \%$ shift in source positioning relative to its diameter. The $\left(\hat{\mathbf{c}_{\mathbf{k}}}, \hat{K}\right)$ version of the algorithm seemed to slightly improve results for most cases - except when $\delta\left(Q_{\text {pos }}\right)=0$ and for some instances in the vessel phantom. This general improvement probably happens because adjusting $K$ can slightly compensate for variations in average energy distribution (caused by beam displacement) around the central area where the structures of interest are. Once more, $\left(\hat{\mathbf{c}_{\mathbf{k}}}, \hat{K}\right)$ only improves estimation up to a certain point, after which it seems to stabilise at a local minimum (data not shown) since the fixed wrong positioning means the model can only explain and compensate the data to a certain extent.

Comparing $\mathbf{R}^{\text {(tubes) }}$ between model-based inversions and linear unmixing shows the former perform significantly better for errors in positioning up to $10 \%$ of the beam diameter. 


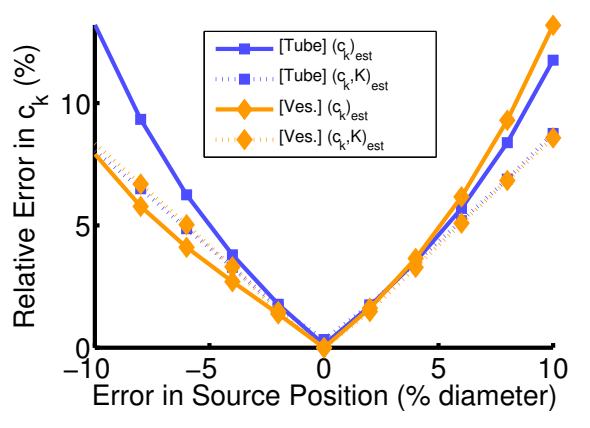

(a)

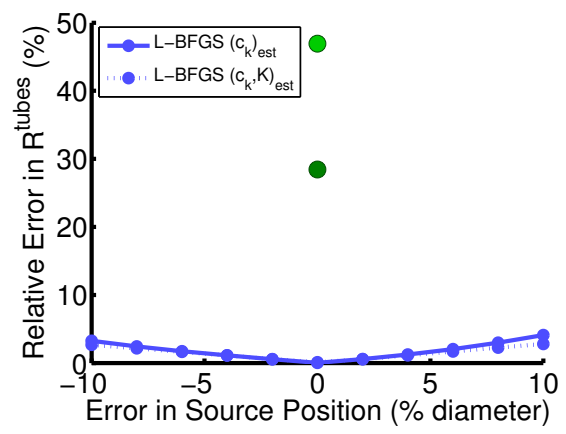

(b)

Figure 7: (a) Relative error in chromophore concentration vs error in source position $Q_{\text {pos }}$. (b) [Tube phantom] Relative error in intra-tube $R$ vs error in source position $Q_{p o s}$. LU - light green; $\mathrm{LU}_{C}$ - dark green.

\subsubsection{Uncertainty on beam diameter}

The beam diameter was varied so that at its largest it would cover the whole lateral range of the domain. $\varepsilon\left(Q_{\text {diam }}\right)=\{-20,-16,-12,-8,-4,0,4,8,12,16,20\} \%$, which corresponds to a diameter ranging from 4 to 6 $\mathrm{mm}$. The source intensity per node was varied so that the total incident fluence remained constant despite the change in diameter. For $\left(\hat{\mathbf{c}_{\mathbf{k}}}\right)$, changes in diameter led to errors in $\mathbf{c}_{\mathbf{k}}$ estimation up to $40 \%$, larger than the error caused by position shifting across the domain. Nevertheless, when applying $\left(\hat{\mathbf{c}_{\mathbf{k}}}, \hat{K}\right)$ the total error in $\mathbf{c}_{\mathbf{k}}$ fell below $2.5 \%$ for all cases. This indicates that actually most of the error in $\left(\hat{\mathbf{c}_{\mathbf{k}}}\right)$ stems more from an erroneous fluence per pixel in the central region rather than from the change in overall fluence spatial distribution that a change in profile diameter produces. Since the tubes are placed quite centrally relative to the beam, and at a reasonable depth, light has become diffuse enough that small variations in diameter have little impact. Robustness to changes in beam positioning and diameter would probably not be as great if the subjects of interest were in the sub-diffuse or collimated regime, or if they were not located quite centrally relative to the source.

As for the estimation of $\mathbf{R}^{\text {tubes }}$, the error is smaller than for $\mathbf{c}_{\mathbf{k}}$. Also, once more model-based inversions far outperform linear unmixing strategies for all studied levels of diameter uncertainty.

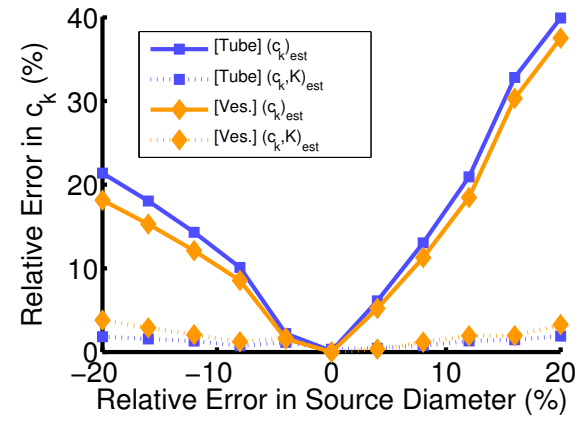

(a)

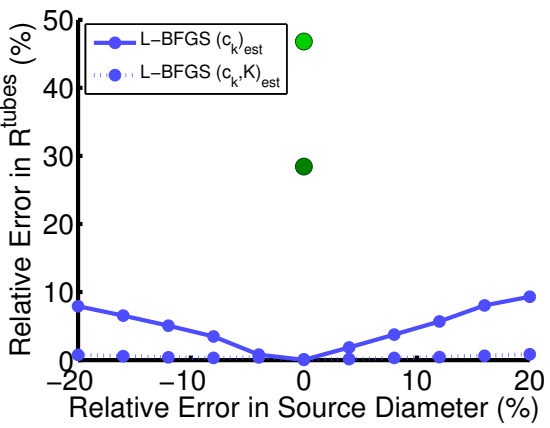

(b)

Figure 8: (a) Relative error in chromophore concentration vs error in source diameter $Q_{\text {diam }}$. (b) [Tube phantom] Relative error in intra-tube $R$ vs error in source diameter $Q_{\text {diam }}$. LU - light green; $\mathrm{LU}_{C}$ - dark green.

In order to have a more visual and comparable sense of the impact of different types of uncertainty, $\mathbf{R}^{\text {(tubes) }}$ of the tube phantom is plotted in Figure 9 for the ground-truth, versus both linear unmixing strategy (LU and $\mathrm{LU}_{c}$ ) outcomes and the L-BFGS- $(\hat{\mathbf{k}})$ algorithm outcomes with parameter uncertainty $|\varepsilon|=20 \%$ (since there are two cases where $|\varepsilon|=20 \%$, the case where $R E\left(\mathbf{c}_{\mathbf{k}}\right)$ was largest is presented). For the case of source positioning, since the parameter error is not relative to itself, the case leading to the worst $R E\left(\mathbf{c}_{\mathbf{k}}\right)$ was plotted, i.e. $\delta\left(Q_{\text {pos }}\right)=10 \% Q_{\text {diam }}$. 


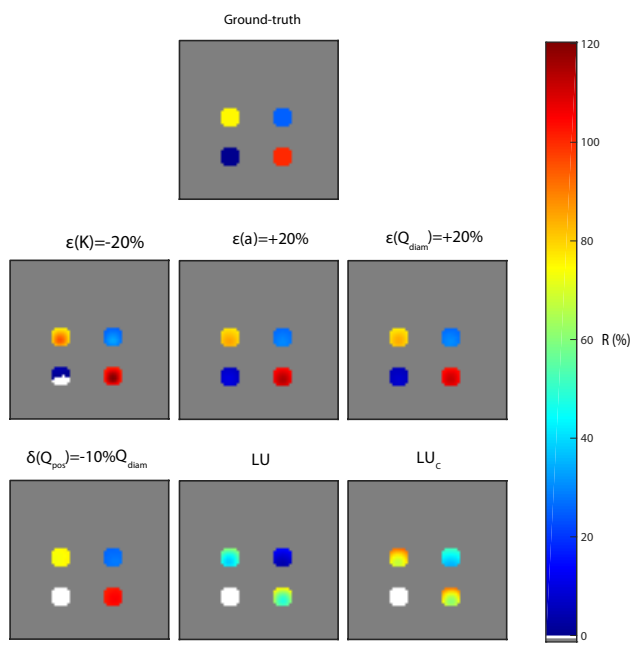

Figure 9: [Tube phantom] Comparison of the inversion outcome in terms of the saturation $R^{\text {tubes }}$, for model-based inversion L-BFGS- $\left(\hat{\mathbf{c}_{\mathbf{k}}}\right)$ with erroneous calibration factor $K$, reduced scattering coefficient $\mu_{s}^{\prime}$, source diameter $Q_{\text {diam }}$ or source positioning $Q_{\text {pos }}$ and for the linear unmixing strategies with and without $1 \mathrm{D}$ background decay correction. White - negative; Gray - background; Darkest blue - zero.

\section{DISCUSSION AND CONCLUSIONS}

Although one advantage of performing in silico studies is that it is possible to assess rigorously the impact the different assumptions made by different inversion approaches has on the parameter estimates, the main goal for the studies presented here was rather different. Here, an in silico study was used to determine the degree to which the accuracy of model-based inversion is sensitive to uncertainty in the input parameters which must be passed to the forward models used in the inversions. The main findings were:

- Experimental uncertainties in a priori fixed parameters - especially calibration factor and scatterer concentration - can affect accuracy of model-based inversions considerably. For all cases, this error is larger when estimating $\mathbf{c}_{\mathbf{k}}$ than when estimating $\mathbf{R}^{\text {tubes }}$, a parameter analogous to $\mathrm{SO}_{2}$;

- Including a global floating scaling parameter in the inversion appears to improve quantification estimates even if the data is originally scaled correctly, probably by absorbing the mean error that different types of parameter uncertainty produce;

- For the case of uncertainty in scattering, including the scatterer concentration as an unknown (with known spectral dependence) further increased the accuracy;

- For realistic levels of experimental uncertainty in model-based input parameters, the studied model-based inversions outperformed linear unmixing approaches (both with and without fluence correction).

It is important to have an understanding of which input parameters are most likely to result in errors in the estimates, as then mitigating measures can be taken. For example, an additional experimental step could be used to obtain this parameter with a higher degree of certainty, or a different physical model that does not require that input could be used, or the parameter may be included as a variable in the inversion (as was done here). ${ }^{2,25}$ If a Bayesian inversion strategy is used, further information on the probability density function of the parameter can be incorporated as prior information, ${ }^{26,27}$ although at potentially considerable computational cost.

\section{ACKNOWLEDGMENTS}

The work presented was supported by a CASE studentship jointly funded by the UK's Engineering and Physical Sciences Research Council and National Physical Laboratory. 


\section{REFERENCES}

[1] Cox, B., Laufer, J. G., Arridge, S. R., and Beard, P. C., "Quantitative spectroscopic photoacoustic imaging: a review," Journal of Biomedical Optics 17(6), 061202 (2012).

[2] Bal, G. and Ren, K., "On multi-spectral quantitative photoacoustic tomography in diffusive regime," Inverse Problems 28(2), 025010 (2012).

[3] Shao, P., Cox, B., and Zemp, R. J., "Estimating optical absorption, scattering, and Grueneisen distributions with multiple-illumination photoacoustic tomography," Applied Optics 50(19), 3145-3154 (2011).

[4] Saratoon, T., Tarvainen, T., Cox, B. T., and Arridge, S. R., "A gradient-based method for quantitative photoacoustic tomography using the radiative transfer equation," Inverse Problems 29(7), 075006 (2013).

[5] Laufer, J., Zhang, E., and Beard, P., "Evaluation of Absorbing Chromophores Used in Tissue Phantoms for Quantitative Photoacoustic Spectroscopy and Imaging," IEEE Journal of Selected Topics in Quantum Electronics 16(3), 600-607 (2010).

[6] van Staveren, H. J., Moes, C. J. M., van Marie, J., Prahl, S. A., van Gemert, M. J., van Staveren, H. J., van Marie, J., and van Gemert, M. J. C., "Light scattering in Intralipid-10\% in the wavelength range of 400-1100 nm.," Applied Optics 30, 4507-14 (Nov. 1991).

[7] Palmer, K. F. and Williams, D., "Optical properties of water in the near infrared," Joural Optical Society of America 64(8), 1107-1110 (1974).

[8] Jacques, S. L., "Optical properties of biological tissues: a review," Physics in Medicine and Biology 58(14), 5007-5008 (2013).

[9] Hall, J., [Guyton and Hall Textbook of Medical Physiology], Guyton Physiology, Elsevier Health Sciences (2010).

[10] Jakubowski, D. B., Cerussi, A. E., Bevilacqua, F., Shah, N., Hsiang, D., Butler, J., and Tromberg, B. J., "Monitoring neoadjuvant chemotherapy in breast cancer using quantitative diffuse optical spectroscopy: a case study," Journal of Biomedical Optics 9(1), 230-238 (2004).

[11] Alexandrakis, G., Rannou, F. R., and Chatziioannou, A., "Tomographic bioluminescence imaging by use of a combined optical-PET (OPET) system: a computer simulation feasibility study," Physics in Medicine ${ }^{6}$ Biology 50(17), 4225-4241 (2005).

[12] Bashkatov, A. N., Genina, E. A., and Tuchin, V. V., "Optical properties of skin, subcutaneous, and muscle tissues: A review," Journal of Innovative Optical Health Sciences 04(01), 9-38 (2011).

[13] Prahl, S., "Omlc tabulated molar extinction coefficient for hemoglobin in water." http://omlc.org/ spectra/hemoglobin/summary.html. Accessed: 2015-08-05.

[14] Prahl, S., "Omlc optical absorption of fat." http://omlc.org/spectra/hemoglobin/summary.html. Accessed: 2016-01-19.

[15] van Veen, R. L., Sterenborg, H., Pifferi, A., Torricelli, A., and Cubeddu, R., "Determination of vis- nir absorption coefficients of mammalian fat, with time- and spatially resolved diffuse reflectance and transmission spectroscopy," in [Biomedical Topical Meeting], Biomedical Topical Meeting, SF4, Optical Society of America (2004).

[16] Schweiger, M. and Arridge, S., "The Toast++ software suite for forward and inverse modeling in optical tomography.," Journal of Biomedical Optics 19(4), 040801 (2014).

[17] Hochuli, R., Beard, P. C., and Cox, B., "Effect of wavelength selection on the accuracy of blood oxygen saturation estimates obtained from photoacoustic images," Proceedings of SPIE 9323, 93231V-8 (2015).

[18] Nocedal, J. and Wright, S. J., [Numerical Optimization], Springer, New York, 2nd ed. (2006).

[19] Cox, B. T., Arridge, S. R., and Beard, P. C., "Gradient-based quantitative photoacoustic image reconstruction for molecular imaging," Proceedings of SPIE 6437, 64371T-64371T-10 (2007).

[20] Soonthornsaratoon, T., Gradient-based methods for quantitative photoacoustic tomography, PhD thesis, University College London, London, UK (2014).

[21] Glatz, J., Deliolanis, N. C., Buehler, A., Razansky, D., and Ntziachristos, V., "Blind source unmixing in multi-spectral optoacoustic tomography.," Optics Express 19(4), 3175-84 (2011).

[22] Hochuli, R., Beard, P. C., and Cox, B., "Accuracy of approximate inversion schemes in quantitative photacoustic imaging," Proceedings of SPIE 8943, 89435V-1 - 89435V-10 (Mar. 2014). 
[23] Zemp, R. J., "Quantitative photoacoustic tomography with multiple optical sources.," Applied Optics 49(18), 3566-72 (2010).

[24] Cox, B. T., Arridge, S. R., and Beard, P. C., "Estimating chromophore distributions from multiwavelength photoacoustic images.," Journal Optical Society of America 26, 443-455 (Feb. 2009).

[25] Bal, G. and Ren, K., "Multi-source quantitative photoacoustic tomography in a diffusive regime," Inverse Problems 27(7), 075003 (2011).

[26] Pulkkinen, A., Cox, B. T., Arridge, S. R., Kaipio, J. P., and Tarvainen, T., "A Bayesian approach to spectral quantitative photoacoustic tomography," Inverse Problems 30(6), 065012 (2014).

[27] Tarvainen, T., Pulkkinen, A., Cox, B. T., Kaipio, J. P., and Arridge, S. R., "Bayesian Image Reconstruction in Quantitative Photoacoustic Tomography," IEEE Transactions on Medical Imaging 32(12), 2287-2298 (2013). 